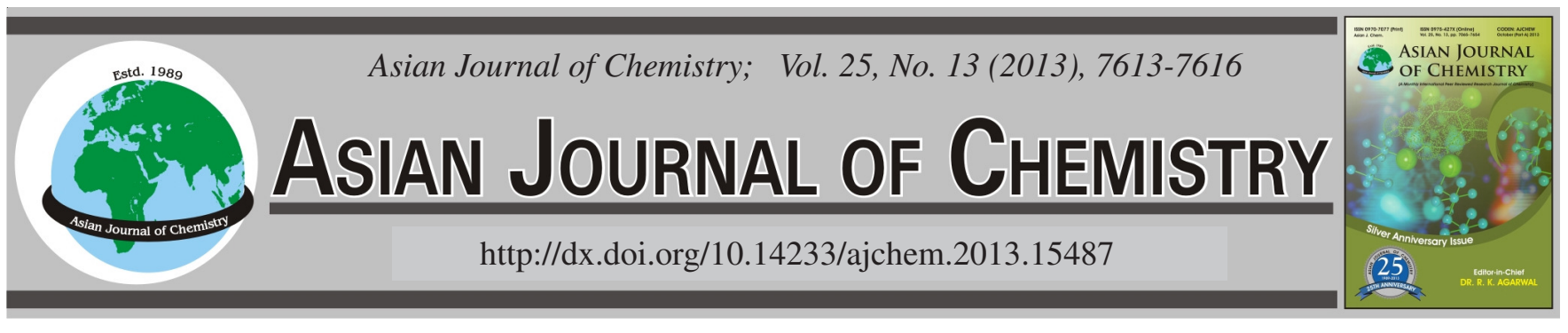

\title{
Accumulation of a Specific Nuclide by Female Common Skete (Feminam Okamejei kenojei spp.)
}

\author{
Hidemitsu Katsura ${ }^{1,2, *}$
}

${ }^{1}$ The United Graduate School of Agricultural Science, Tokyo University of Agriculture \& Technology, Saiwai-Cho 3-Chome, Fuchu-Shi, Tokyo 183-8509, Japan

${ }^{2}$ Department of Ocean Sciences, Faculty of Marine Science, Tokyo University of Marine Science and Technology, Konan 4-Chome, Minato-Ku, Tokyo 108-8477, Japan

*Corresponding author: Fax: +81 467 541383; Tel: +81 467 545869; E-mail: hi@katsura.dk

Key Words: Accumulation, Nuclide, Okamejei kenojei spp.

ᄂ - - - - - - - - - - - - - - - - - - - - - - - - -

\section{INTRODUCTION}

Tokyo Electric Power Fukushima Dai-Ichi Nuclear Power Station in Futaba-Gun, Fukushima-Ken, Japan was destroyed due to a magnitude 9 earthquake in the ocean northeast of the Island of Honshu in Japan that produced a historical Tsunami on 11 March 2011. Due to a nuclear meltdown, hydrogen explosions damaged buildings housing reactors, cooling water from the reactor core was contaminated and huge amounts of radioisotopes were released into the atmosphere and marine waters. Currently, offshore fishing in Fukushima-ken, Japan is prohibited because the majority of fish still contain amounts of radioisotopes that exceed the Japanese Standard Value ${ }^{1-4}$. Therefore, the Japanese Government has measured the amounts of radioisotopes in fish offshore the neighbouring regions around the destroyed reactor and across the Exclusive Economic Zone (EEZ) of Japan. Some of these measured data are frequently released to the public on a website by the Japanese Government. However, these data do not contain individual fish data, including fish weight, sex, length and collecting detail position. However, Tokyo University of Marine
Science and Technology independently measured the amounts of radioisotopes found in fish offshore of Iwaki-Shi, located south of the destroyed Nuclear Power Station in FukushimaKen, Japan in September and November 2012. The data collected by Tokyo University of Marine Science and Technology included detailed individual fish data, such as fish weight, sex, length and collecting detail position. Using the original data collected in September and November 2012, a negative linear relationship between fish weight and ${ }^{137} \mathrm{Cs} /{ }^{134} \mathrm{Cs}$ ratio was found in feminam Okamejei kenojei spp.

\section{EXPERIMENTAL}

Okamejei kenojei spp. (English Name: Common skete; Japanese Name: Komon Kasube) fish were caught by Trawl Net on 19 September 2012 and 22 Novemeber 2012 offshore of Iwaki-Shi, Fukushima-Ken, Japan. The edible parts of the fish were minced and packed into a $100 \mathrm{~mL}$ U-8 container. The concentrations of radioisotopes ${ }^{134} \mathrm{Cs}$ and ${ }^{137} \mathrm{Cs}$ in the fish meat were measured using a germanium semiconductor detector (SEIKO EG \& G, model GEM20-70) by IDEA Consultants Inc., Tokyo, Japan ${ }^{5}$. 


\section{RESULTS AND DISCUSSION}

Japanese Government revealed that fallout amounts of ${ }^{134} \mathrm{Cs}$ and ${ }^{137} \mathrm{Cs}$ radioisotopes in Futaba-Gun, Fukushima-Ken, Japan in March 2011 were $3100000 \mathrm{MBq} / \mathrm{km}^{2}$ and 3340000 $\mathrm{MBq} / \mathrm{km}^{2}$, respectively, due to the destruction of the Tokyo Electric Power Fukushima Dai-Ichi Nuclear Power Station in Futaba-Gun, Fukushima-Ken, Japan on 11 March $2011^{6}$. The half-life of ${ }^{134} \mathrm{Cs}$ is 2.0648 years (754.17 days) and the halflife of ${ }^{137} \mathrm{Cs}$ is 30.1671 years (11018.5 days). Therefore, if contaminated fish directly accumulated ${ }^{134} \mathrm{Cs}$ and ${ }^{137} \mathrm{Cs}$ without any physiological effects or environmental factors, the ${ }^{137} \mathrm{Cs} /$ ${ }^{134} \mathrm{Cs}$ ratio in the bodies of all fish offshore of Fukushima-Ken should be similar to the ratios in Table-1 and Fig. 1. The ${ }^{137} \mathrm{Cs} /$ ${ }^{134} \mathrm{Cs}$ ratio in all contaminated fish offshore of FukushimaKen on 19 September 2012 should be 1.73727244219614 and the ${ }^{137} \mathrm{Cs} /{ }^{134} \mathrm{Cs}$ ratio on 22 November 2012 should be 1.8351234381334 . However, the measured ${ }^{137} \mathrm{Cs} /{ }^{134} \mathrm{Cs}$ ratio in contaminated fish was very different, indicating variation in the ${ }^{137} \mathrm{Cs} /{ }^{134} \mathrm{Cs}$ ratio in fish.

Okamejei kenojei spp. was the major fish species offshore of Fukushima-Ken, Japan, in September and November 2012. The concentrations of ${ }^{134} \mathrm{Cs}$ and ${ }^{137} \mathrm{Cs}$ and the ${ }^{137 / 134} \mathrm{Cs}$ ratio of Okamejei kenojei spp. offshore of Iwaki-Shi, Fukushima-Ken is shown in Table-2 for September 2012 and Table-3 for November 2012.

Okamejei kenojei spp. body weight $v$ s. concentrations of ${ }^{134} \mathrm{Cs},{ }^{137} \mathrm{Cs}$ and the ${ }^{137} \mathrm{Cs} /{ }^{134} \mathrm{Cs}$ ratio is shown in Fig. 2 for September 2012 and Fig. 5 for November 2012. However, no correlations were found between fish body weight and concentrations of ${ }^{134} \mathrm{Cs},{ }^{137} \mathrm{Cs}$ and the ${ }^{137} \mathrm{Cs} /{ }^{134} \mathrm{Cs}$ ratio.

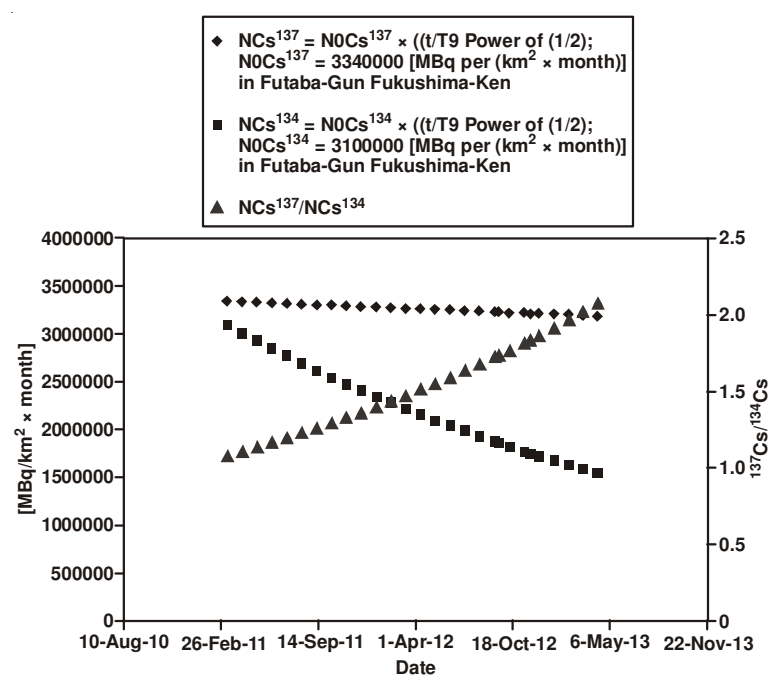

Fig. 1. Ratios ${ }^{137} \mathrm{Cs} /{ }^{134} \mathrm{Cs}$ from 11-March-2011 in Futaba-Gun, Fukushimaken, Japan

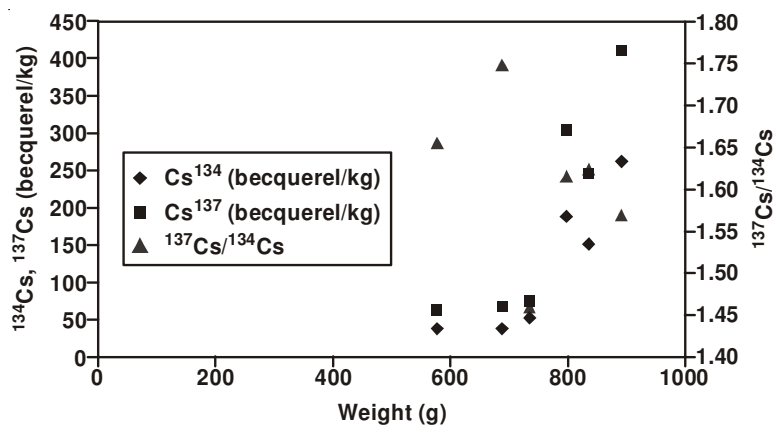

Fig. 2. Okamejei kenojei spp. [Common Skete spp.] fish body weight vs. concentration of ${ }^{134} \mathrm{Cs}$, ${ }^{137} \mathrm{Cs}$ and ${ }^{137} \mathrm{Cs} /{ }^{134} \mathrm{Cs}$ in September 2012

\begin{tabular}{|c|c|c|c|c|c|c|c|c|c|c|}
\hline \multicolumn{11}{|c|}{$\begin{array}{l}\text { TABLE-1 } \\
\text { RATIOS }{ }^{137} \mathrm{Cs} /{ }^{134} \mathrm{Cs} \text { FROM 11-MARCH-2011 IN FUTABA-GUN, FUKUSHIMA-KEN, JAPAN }\end{array}$} \\
\hline 1 & 2 & 3 & 4 & 5 & 6 & 7 & 8 & 9 & 10 & 11 \\
\hline 11-Mar-11 & - & 11018.5 & - & - & 3340000 & 754.17 & - & - & 3100000 & 1.077419355 \\
\hline 11-Apr-11 & 31 & 11018.5 & 0.002813450 & 0.998051765 & 3333492.896 & 754.17 & 0.041104791 & 0.971910390 & 3012922.210 & 1.106398594 \\
\hline 11-Мау-11 & 61 & 11018.5 & 0.005536144 & 0.996169991 & 3327207.769 & 754.17 & 0.080883620 & 0.945478384 & 2930982.989 & 1.135184947 \\
\hline 11-Jun-11 & 92 & 11018.5 & 0.008349594 & 0.994229218 & 3320725.588 & 754.17 & 0.121988411 & 0.918920265 & 2848652.821 & 1.165717901 \\
\hline 11-Jul-11 & 122 & 11018.5 & 0.011072288 & 0.992354651 & 3314464.533 & 754.17 & 0.161767241 & 0.893929374 & 2771181.060 & 1.196047628 \\
\hline 11-Sep-11 & 184 & 11018.5 & 0.016699188 & 0.988491738 & 3301562.404 & 754.17 & 0.243976822 & 0.844414453 & 2617684.805 & 1.261252843 \\
\hline 11-Oct-11 & 214 & 11018.5 & 0.019421881 & 0.986627988 & 3295337.480 & 754.17 & 0.283755652 & 0.821449817 & 2546494.434 & 1.294068205 \\
\hline 11-Nov-11 & 245 & 11018.5 & 0.022235331 & 0.984705805 & 3288917.389 & 754.17 & 0.324860443 & 0.798375613 & 2474964.399 & 1.328874626 \\
\hline 11-Dec-11 & 275 & 11018.5 & 0.024958025 & 0.982849194 & 3282716.308 & 754.17 & 0.364639272 & 0.776663046 & 2407655.441 & 1.363449375 \\
\hline 11-Jan-12 & 306 & 11018.5 & 0.027771475 & 0.980934373 & 3276320.806 & 754.17 & 0.405744063 & 0.754846884 & 2340025.340 & 1.400121935 \\
\hline 11-Feb-12 & 337 & 11018.5 & 0.030584925 & 0.979023283 & 3269937.764 & 754.17 & 0.446848854 & 0.733643529 & 2274294.941 & 1.437780872 \\
\hline 11-Mar-12 & 366 & 11018.5 & 0.033216863 & 0.977238859 & 3263977.789 & 754.17 & 0.485301722 & 0.714347657 & 2214477.737 & 1.473926667 \\
\hline 11-May-12 & 427 & 11018.5 & 0.038753006 & 0.973496025 & 3251476.725 & 754.17 & 0.566185343 & 0.675400268 & 2093740.831 & 1.552950908 \\
\hline 11-Jun-12 & 458 & 11018.5 & 0.041566456 & 0.971599427 & 3245142.085 & 754.17 & 0.607290134 & 0.656428538 & 2034928.468 & 1.594720470 \\
\hline 11-Jul-12 & 488 & 11018.5 & 0.044289150 & 0.969767527 & 3239023.539 & 754.17 & 0.647068963 & 0.638576354 & 1979586.697 & 1.636212015 \\
\hline 11-Aug-12 & 519 & 11018.5 & 0.047102600 & 0.967878192 & 3232713.161 & 754.17 & 0.688173754 & 0.620638993 & 1923980.879 & 1.680221043 \\
\hline 11-Sep-12 & 550 & 11018.5 & 0.049916050 & 0.965992538 & 3226415.077 & 754.17 & 0.729278545 & 0.603205486 & 1869937.007 & 1.725413778 \\
\hline 19-Sep-12 & 558 & 11018.5 & 0.050642102 & 0.965506514 & 3224791.757 & 754.17 & 0.739886233 & 0.598786569 & 1856238.365 & 1.737272442 \\
\hline 20-Sep-12 & 559 & 11018.5 & 0.050732858 & 0.965445778 & 3224588.900 & 754.17 & 0.741212194 & 0.598236486 & 1854533.106 & 1.738760495 \\
\hline 11-Oct-12 & 580 & 11018.5 & 0.052638744 & 0.964171209 & 3220331.840 & 754.17 & 0.769057374 & 0.586800752 & 1819082.332 & 1.770305710 \\
\hline 11-Nov-12 & 611 & 11018.5 & 0.055452194 & 0.962292778 & 3214057.877 & 754.17 & 0.810162165 & 0.570317748 & 1767985.019 & 1.817921443 \\
\hline 22-Nov-12 & 622 & 11018.5 & 0.056450515 & 0.961627117 & 3211834.572 & 754.17 & 0.824747736 & 0.564580914 & 1750200.834 & 1.835123438 \\
\hline 23-Nov-12 & 623 & 11018.5 & 0.056541271 & 0.961566626 & 3211632.530 & 754.17 & 0.826073697 & 0.564062254 & 1748592.988 & 1.836695304 \\
\hline
\end{tabular}




\begin{tabular}{|c|c|c|c|c|c|c|c|}
\hline \multicolumn{8}{|c|}{$\begin{array}{l}\text { TABLE-2 } \\
\text { CONCENTRATION OF }{ }^{134} \text { Cs AND }{ }^{137} \text { Cs AND RATIOS }{ }^{137 / 134} \text { Cs OF Okamejei kenojei spp. } \\
\text { IN SEPTEMBER } 2012 \text { IN OFFSHORE IWAKI-SHI, FUKUSHIMA-KEN }\end{array}$} \\
\hline $\begin{array}{l}\text { Sampling } \\
\text { date }\end{array}$ & Way of sampling & $\begin{array}{c}\text { Size }\left(\mathrm{cm}^{2}\right) \\
(\text { Vertical } \times \text { Horizontal })\end{array}$ & $\begin{array}{l}\text { Weight } \\
(\mathrm{g})\end{array}$ & Remarks & $\begin{array}{c}{ }^{134} \mathrm{Cs} \\
\text { (becquerel/kg) }\end{array}$ & $\begin{array}{c}{ }^{137} \mathrm{Cs} \\
\text { (becquerel/kg) }\end{array}$ & ${ }^{137} \mathrm{Cs} /{ }^{134} \mathrm{Cs}$ \\
\hline 19-Sep-2012 & Trawl (Net) [Dragneta Trawlnet] & $49.0 \times 34.0$ & 797.3 & Female & 189 & 305 & 1.613756614 \\
\hline 19-Sep-2012 & Trawl (Net) [Dragneta Trawlnet] & $41.0 \times 29.0$ & 577.3 & Male & 36.8 & 60.9 & 1.654891304 \\
\hline 19-Sep-2012 & Trawl (Net) [Dragneta Trawlnet] & $49.0 \times 34.0$ & 891.1 & Female & 262 & 411 & 1.568702290 \\
\hline 19-Sep-2012 & Trawl (Net) [Dragneta Trawlnet] & $42.0 \times 32.0$ & 734.5 & Male & 50.9 & 74.2 & 1.457760314 \\
\hline 19-Sep-2012 & Trawl (Net) [Dragneta Trawlnet] & $52.5 \times 35.5$ & 835.4 & Male & 151 & 245 & 1.622516556 \\
\hline 19-Sep-2012 & Trawl (Net) [Dragneta Trawlnet] & $44.0 \times 32.0$ & 688.2 & Female & 37.5 & 65.5 & 1.746666667 \\
\hline
\end{tabular}

TABLE-3

CONCENTRATION OF ${ }^{134} \mathrm{Cs}$ AND ${ }^{137} \mathrm{Cs}$ AND ratios ${ }^{137 / 134} \mathrm{Cs}$ OF Okamejei kenojei spp.

IN NOVEMBER 2012 IN OFFSHORE IWAKI-SHI, FUKUSHIMA-KEN

\begin{tabular}{|c|c|c|c|c|c|c|c|}
\hline $\begin{array}{l}\text { Sampling } \\
\text { date }\end{array}$ & Way of sampling & $\begin{array}{c}\text { Size }\left(\mathrm{cm}^{2}\right) \\
(\text { Vertical } \times \text { Horizontal })\end{array}$ & $\begin{array}{l}\text { Weight } \\
\text { (g) }\end{array}$ & Remarks & $\begin{array}{c}{ }^{134} \mathrm{Cs} \\
\text { (becquerel } / \mathrm{kg} \text { ) }\end{array}$ & $\begin{array}{c}{ }^{137} \mathrm{Cs} \\
\text { (becquerel/kg) }\end{array}$ & ${ }^{137} \mathrm{Cs} /{ }^{134} \mathrm{Cs}$ \\
\hline 22-Nov-2012 & Trawl (Net) [Dragneta Trawlnet] & $45.0 \times 32.1$ & 702.2 & Male & 36.6 & 66.6 & 0.54954955 \\
\hline 22-Nov-2012 & Trawl (Net) [Dragneta Trawlnet] & $56.1 \times 39.5$ & 1186.5 & Female & 59.2 & 96.2 & 0.615384615 \\
\hline 22-Nov-2012 & Trawl (Net) [Dragneta Trawlnet] & $53.2 \times 37.0$ & 1025.9 & Male & 43.4 & 66.4 & 0.653614458 \\
\hline 22-Nov-2012 & Trawl (Net) [Dragneta Trawlnet] & $46.0 \times 33.0$ & 725.2 & Female & 49.9 & 72.8 & 0.68543956 \\
\hline 22-Nov-2012 & Trawl (Net) [Dragneta Trawlnet] & $43.7 \times 32.7$ & 742.3 & Male & 50.9 & 92.0 & 0.55326087 \\
\hline 22-Nov-2012 & Trawl (Net) [Dragneta Trawlnet] & $43.6 \times 31.0$ & 624.0 & Male & 30.8 & 49.5 & 0.622222222 \\
\hline 22-Nov-2012 & Trawl (Net) [Dragneta Trawlnet] & $43.9 \times 30.5$ & 614.6 & Female & 25.3 & 47.5 & 0.532631579 \\
\hline 22-Nov-2012 & Trawl (Net) [Dragneta Trawlnet] & $48.8 \times 34.0$ & 809.9 & Male & 26.9 & 47.0 & 0.572340426 \\
\hline
\end{tabular}

Male Okamejei kenojei spp. body weight $v s .{ }^{137} \mathrm{Cs} /{ }^{134} \mathrm{Cs}$ ratio is shown in Fig. 3 for September 2012 and Fig. 6 for November 2012. However, no correlations were found between fish body weight and the ${ }^{137} \mathrm{Cs} /{ }^{134} \mathrm{Cs}$ ratio.

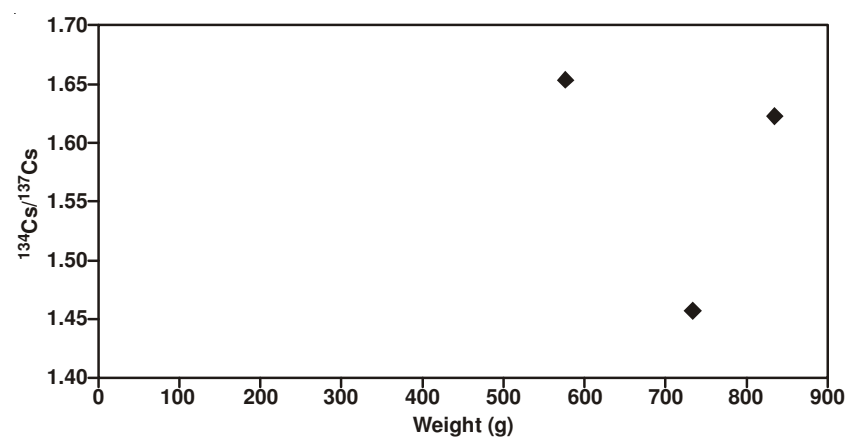

Fig. 3. Male Okamejei kenojei spp. [Common Skete spp.] fish body weight vs. ${ }^{137} \mathrm{Cs} /{ }^{134} \mathrm{Cs}$ in September 2012

Female Okamejei kenojei spp. body weight $v s .{ }^{137} \mathrm{Cs} /{ }^{134} \mathrm{Cs}$ ratio is shown in Fig. 4 for September 2012 and Fig. 7 for November 2012. A negative linear correlation between fish body weight and the ${ }^{137} \mathrm{Cs} /{ }^{134} \mathrm{Cs}$ ratio was found. Therefore, this result suggests that feminam Okamejei kenojei spp. have the ability to accumulate a specific nuclide.

Okamejei kenojei spp. have the ability to detect weak electric signals and they receive electrical information on the positions of their prey, the drift of ocean currents ${ }^{7-9}$ and their magnetic compass headings. Radioisotopes ${ }^{134} \mathrm{Cs}$ and ${ }^{137} \mathrm{Cs}$ are $\beta$-emission nuclides and emit $\beta$-rays and $\gamma$-rays ${ }^{10,11}$. A $\beta$-ray is an electron beam or electric current and a $\gamma$-ray is a radio wave or electromagnetic wave of small wavelength. A major food source for Okamejei kenojei spp. is Metapenaeopsis dalei (Japanese Name: KISHI EBI) ${ }^{12}$ and the radioisotope concentration in Metapenaeopsis dalei varied. The radioisotope concentration in Metapenaeopsis dalei depended on individual bodies

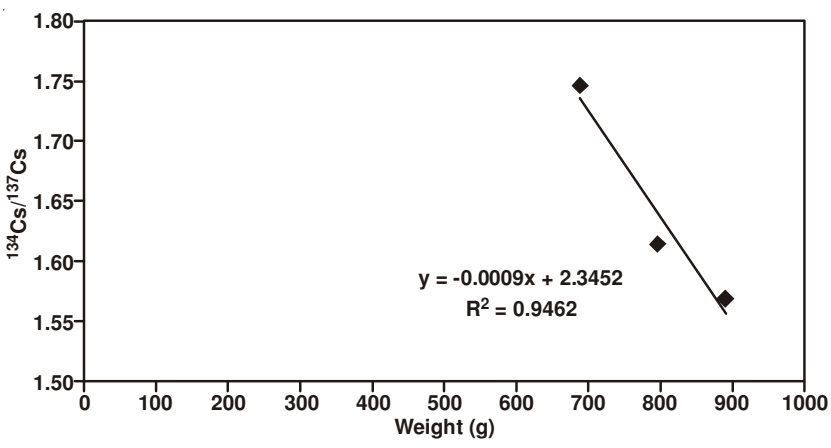

Fig. 4. Female Okamejei kenojei spp. [Common Skete spp.] fish body weight vs. ${ }^{137} \mathrm{Cs} /{ }^{134} \mathrm{Cs}$ in September 2012

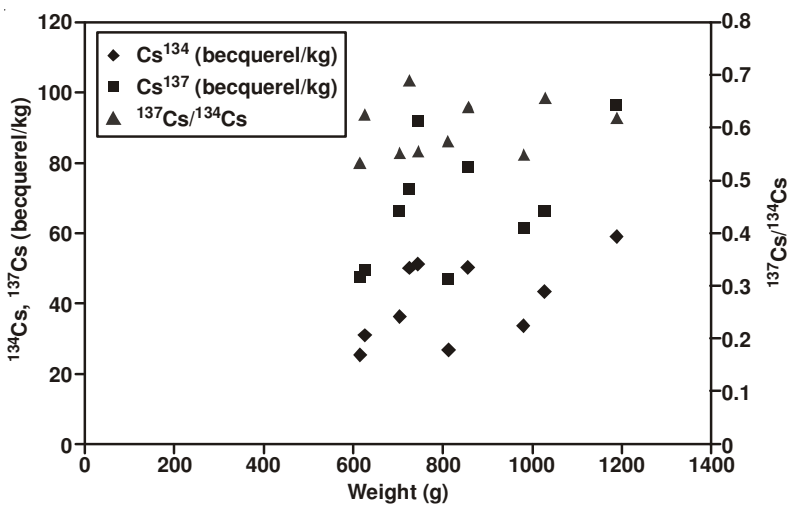

Fig. 5. Okamejei kenojei spp. [Common Skete spp.] fish body weight $v s$. concentration of ${ }^{134} \mathrm{Cs},{ }^{137} \mathrm{Cs}$ and ${ }^{137} \mathrm{Cs} /{ }^{134} \mathrm{Cs}$ in November 2012

and the average radioisotope concentration in individual bodies was not meaningful to this investigation ${ }^{13,14}$. If the Metapenaeopsis dalei radioisotope ratios changed depending on the body weight of feminam Okamejei kenojei spp., the negative linear correlation between Okamejei kenojei spp. body weight and the ${ }^{137} \mathrm{Cs} /{ }^{134} \mathrm{Cs}$ ratio could be explained by the ability of Okamejei kenojei spp. to detect weak electric signals. 


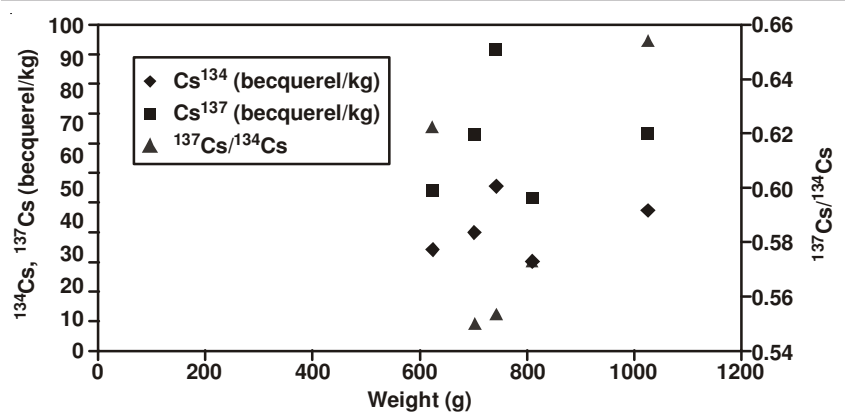

Fig. 6. Male Okamejei kenojei spp. [Common Skete spp.] fish body weight vs. ${ }^{137} \mathrm{Cs} /{ }^{134} \mathrm{Cs}$ in November 2012

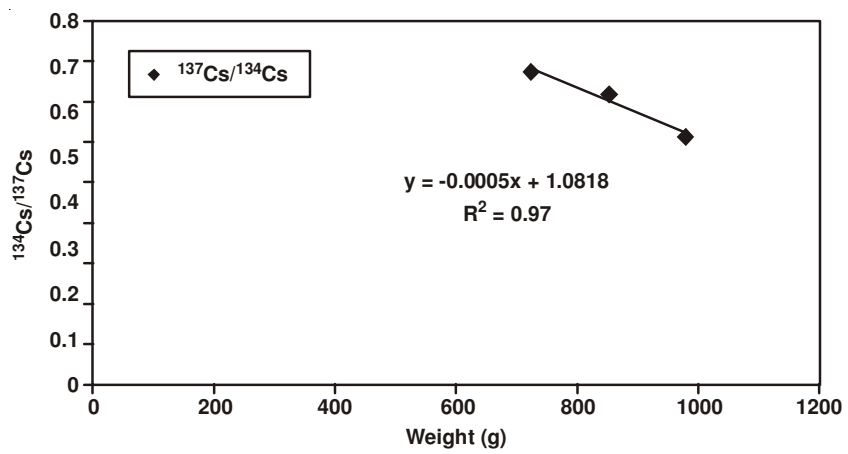

Fig. 7. Female Okamejei kenojei spp. [Common Skete spp.] fish body weight vs. ${ }^{137} \mathrm{Cs} /{ }^{134} \mathrm{Cs}$ in November 2012

\section{Conclusion}

- A negative linear correlation between feminam Okameje $i$ kenojei spp. body weight and the ${ }^{137} \mathrm{Cs} /{ }^{134} \mathrm{Cs}$ ratio was identified (Fig. 4 and 7).

- The methodology in this study has the ability to detect specific $\beta$-ray emissions by feminam Okamejei kenojei spp. Furthermore, this methodology can be used to identify other species that accumulate specific radioisotopes.

- Physical methods such as ultracentrifugation and diffusion have been used to obtain higher concentration nuclides (e.g. $\left.{ }^{235} \mathrm{U}\right)$. However, this study suggests that we do not need these physical methods to accumulate higher concentrations of specific radioisotopes ${ }^{15}$.

\section{ACKNOWLEDGEMENTS}

The author thanks Ms. ARIKI Mizuki, Ms. GORAI Myu, Ms. HENMI Chikako, Mr. KASE Ryota, Mr. NAKAGAWA Takuro and Mr. MYOUSE Hiroshi, undergraduate students at the Tokyo University of Marine Science \& Technology (TUMST); Ms. SHIMOMURA Yukiko, Mr. HASEGAWA Kouhei and Mr. WATANABE Hayato, graduate students at TUMST; Ph.D. Candidate GIRAULT Mathias and Ph.D. Candidate MATSUMOTO Akira at TUMST; Mr. SATO Katsuhiro, Research Scientist, TUMST; Ms. Yoshizaki Kae and Ms. HONDA Tomoko, secretary to Professor ARAKAWA Hisayuki, TUMST; Dr. WAKABAYASHI Kaori, Doctoral Research Scientist, TUMST; Dr. NORMAN Chris, Adjunct Professor, TUMST; Dr. AKIYAMA Seiji, Assistant Professor, TUMST; Dr. UCHIDA Keiichi, Assistant Professor, TUMST; Dr. ARAKAWA Hisayuki, Professor, TUMST and Dr. MORINAGA Tsutomu, Emeritus Professor TUMST for their assistance. This study was supported by the Research Thrust Fund for fiscal year 2012 of the Ministry of the Environment, Japan, for "Study of the diffusion process of radioisotope materials from the destroyed nuclear power plant in offshore ecosystems in Fukushima Ken (Prefecture), Japan [HEISEI 24 NENDO KANKYOUSHOU SOUGOU KENKYUU SUISHINHI].

\section{REFERENCES}

1. S.-I. Tanaka, Proc. Jpn. Acad., Ser. B, 88, 471 (2012).

2. Y. Nemoto, S. Shimamura and S. Igarashi, Nippon Suisan Gakkaishi, 78, 514 (2012)

3. O. Takeo, Radiat. Res., 177, 1 (2012).

4. Japanese Governmental Official Bulletin On-Line (2012). http:// www.gov-online.go.jp/useful/article/201204/3.html

5. M. Tomiaki, N. Hiroyuki, N. Masashi, O. Kimihito and S. Yoshimichi, J. Food Hygien. Soc. Japan, 53, 177 (2012).

6. Ministry of Education, Culture, Sports, Science and Technology (2011). Monitoring information of environmental radioactivity level, Reading of environmental radioactivity level by prefecture [Fallout], March, 2011, [Announcement at 14 o'clock July 29, 2011], [Corrected data on September 28, 2011], Additional date on December 14, 2011, (Accessed:19 July 2011) http://radioactivity.mext.go.jp/old/ja/ monitoring_by_prefecture_fallout/2011/03/1060_03_gekkan_2.pdf

7. A.J. Kalmijn, Science, 218, 916 (1982).

8. G. von der Emde, S. Schwarz, L. Gomez, R. Budelli and K. Grant, Nature, 395, 890 (1998).

9. R.W. Murray, J. Exp. Biol., 39, 119 (1962).

10. J.L. Wolfson, Can. J. Phys., 34, 256 (1956).

11. S. Hellstrom and D. Brune, Nukleonik, 6, 174 (1964).

12. H. Sakaji, Fisheries Sci., 67, 444 (2001).

13. P. McDonald, M.S. Baxter and E.M. Scott, J. Environ. Radioact., 32, 67 (1996).

14. H. Katsura, Asian J. Chem., 25, 2135 (2013).

15. R. Pannier, Haus der Technik-Vortrag Veröffentlichungen, 214, 15 (1982). 